\title{
Functional Connectivity Modulations in Resting State and Offset Analgesia in Chronic Pain Patients: an fMRI Study
}

\section{Tianjiao Li, Eri Ikeda, Hiroyuki Kobinata, Shuo Zhang, Koshi Makita, Jiro Kurata* \\ Dept of Anesthesiology, Graduate School of Medical and Dental Sciences, Tokyo Medical and Dental University, Tokyo, Japan}

\section{Background \& Aims}

Chronic pain, often lacking diagnostic peripheral pathology, has been attributed to cerebral dysfunction from recent neuroimaging findings. Here we used functional magnetic resonance imaging (fMRI) to seek for dynamic alterations in cerebral networks between no-pain and phasic-pain conditions in chronic pain patients with various etiologies. Specifically, we made a comparison between chronic pain patients (CP) and healthy controls (HC) in 1) whole-brain functional connectivity (FC) during resting-state, and 2) dynamic FC changes during offset analgesia (OA)/ sham paradigms by thermal pain stimulus known to trigger endogenous pain modulation.

Methods

Subjects: $16 \mathrm{CP}$ and age-, gender-matched $16 \mathrm{HC}$ subjects; all were righthanded, free from caffeine or alcohol $24 \mathrm{~h}$ before the experiment.

\begin{tabular}{|l|l|}
\hline Disease & No. of CP \\
\hline Fibromyalgia & $\mathbf{7}$ \\
\hline Chronic low back pain & 2 \\
\hline $\begin{array}{l}\text { Complex regional pain } \\
\text { syndrome }\end{array}$ & $\mathbf{2}$ \\
\hline Limb pain & $\mathbf{I}$ \\
\hline Phantom limb pain & $\mathbf{I}$ \\
\hline Cervical syndrome & $\mathbf{I}$ \\
\hline Sciatica & $\mathbf{I}$ \\
\hline Migraine & $\mathbf{I}$ \\
\hline
\end{tabular}

\begin{tabular}{|l|l|}
\hline Medication Treatments & No. of CP \\
\hline Tricyclic Antidepressant Drugs & $\mathbf{1 3}$ \\
\hline $\begin{array}{l}\text { Anticonvulsant and } \\
\text { Antiepileptic Drugs }\end{array}$ & $\mathbf{1 I}$ \\
\hline $\begin{array}{l}\text { Non-Steroidal Anti- } \\
\text { Inflammatory Drugs }\end{array}$ & $\mathbf{6}$ \\
\hline Anti-Anxiety Drugs & $\mathbf{5}$ \\
\hline Tramadol & $\mathbf{3}$ \\
\hline $\begin{array}{l}\text { Serotonin-Noradrenalin } \\
\text { Reuptake Inhibitors }\end{array}$ & $\mathbf{2}$ \\
\hline
\end{tabular}

Behavioral Measurements: Current pain intensity by visual analog scale (VAS), short-form McGill pain questionnaire (SF-MPQ), PainDETECT, Pain Catastrophizing Scale (PCS), and Beck Depression Inventory (BDI).

\begin{tabular}{|c|c|c|c|}
\hline Measurements & HC (Mean \pm SD) & CP (Mean \pm SD) & P-value \\
\hline Sex $(F / M)$ & $8 F / 8 M$ & $7 F / 9 M$ & 0.733 \\
\hline Age $(y r)$ & $42.13 \pm 12.54$ & $42.06 \pm 10.96$ & 0.988 \\
\hline VAS & $0.06 \pm 0.25$ & $51.50 \pm 27.13$ & $<0.00 I^{*}$ \\
\hline PainDETECT & $2.13 \pm 3.96$ & $17.31 \pm 8.26$ & $<0.001^{*}$ \\
\hline SF-MPQ & $0.19 \pm 0.54$ & $15.13 \pm 9.38$ & $<0.00 I^{*}$ \\
\hline PCS & $4.81 \pm 7.37$ & $32.00 \pm 12.05$ & $<0.001^{*}$ \\
\hline BDI & $3.38 \pm 4.86$ & $18.5 \pm 12.90$ & $<0.00 I^{*}$ \\
\hline
\end{tabular}

Noxious Thermal Stimulus

1) Thermal stimulus given on the left volar forearm by a Peltier-type thermal stimulator (PATHWAY, Medoc, Israel) at ramp rates of $56^{\circ} \mathrm{C} / \mathrm{s}$ (up) and $6^{\circ} \mathrm{C} / \mathrm{s}$ (down)

2) Baseline temperature: $32^{\circ} \mathrm{C} . \mathrm{T} 1,46^{\circ} \mathrm{C}$-stimulus $(5 \mathrm{~s}) ; \mathrm{T} 2,47^{\circ} \mathrm{C}$ stimulus (5 s); T3, $46^{\circ} \mathrm{C}$-stimulus (20 s). Offset, Constant and Short ${ }^{\circ}$ conditions were pseudo-randomly applied 3 times for each.

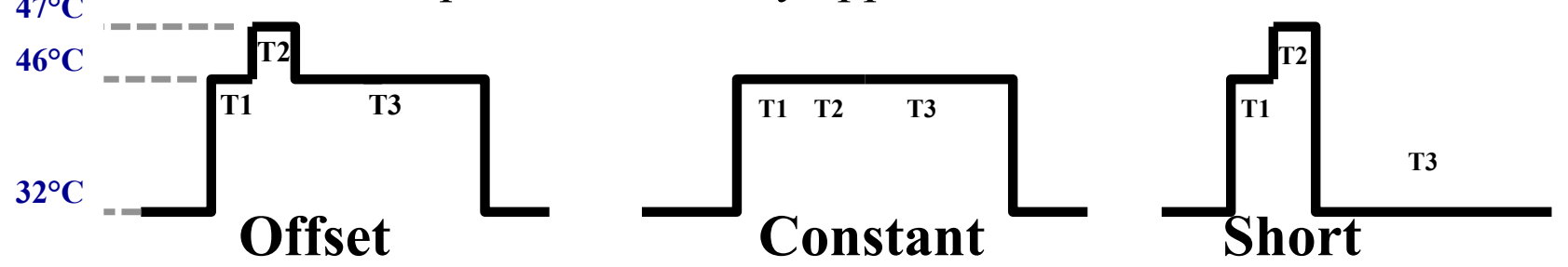

Resting-state: Subjects were required to keep eyes open for $5 \mathrm{~min}$.

MRI scanner: 3T MRI scanner (GE Healthcare, UK); we got gradientecho echo-planar MRI images during thermal stimuli and resting-state, and a high-resolution anatomical image.

Data Analysis:

1) SPM12 (Wellcome Trust Centre for Neuroimaging, UK) and CONN Toolbox were used for analysis of images.

2) $\mathrm{OA}$ effects was examined by $\mathrm{FC}$ contrasts during $\mathrm{T} 3$ at OffsetConstant and Offset-Short

3) ROI-ROI analysis and gPPI analysis were used to investigate FC modulations across different states and conditions with age and gender as covariates.

4) SPSS 23.0 (IBM, USA) was used to examine correlations between FC alterations and behavioral measurements in both $\mathrm{CP}$ and $\mathrm{HC}$ groups.

References

Hermans L, et al, Pain Physician 19(2016):307-326

Grill JD, et al, Journal of Neurophysiology, 2002; 87:2205-2208

Yelle MD, et al, J Pain 2008;134: 174-186

Oudejans LC, et al. J Pain, 2015; 156(12): 2521-2527
Results

A. Connectivity within the default mode network was decreased in $\mathrm{CP}$ compared with $\mathrm{HC}$.

MPFC: middle prefrontal cortex
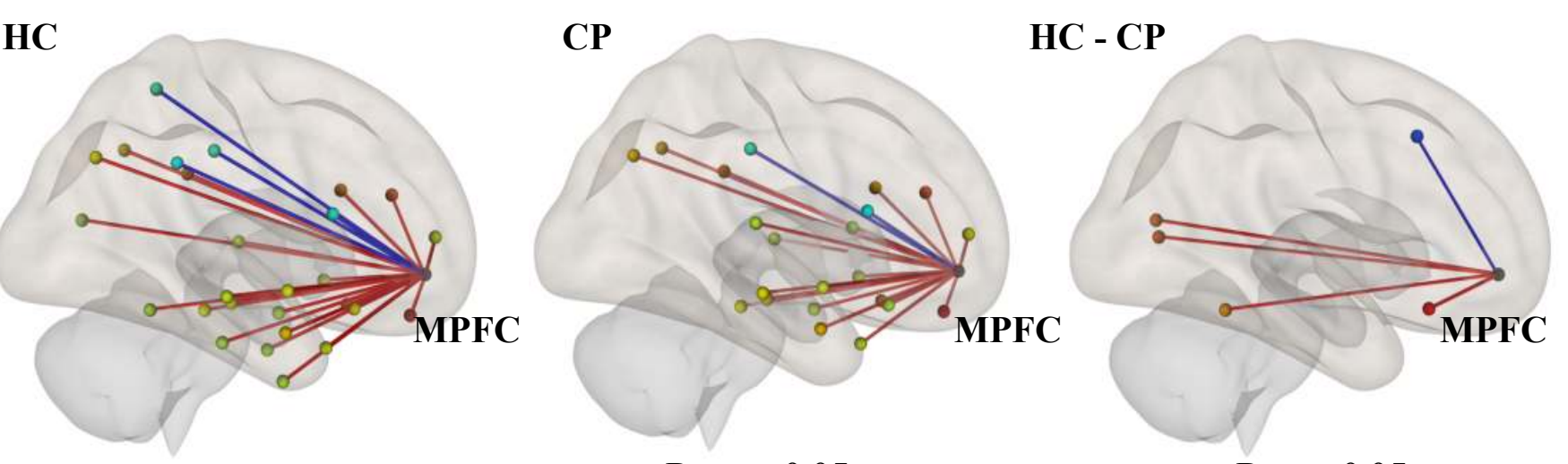

$\mathrm{P}_{\mathrm{FDR}}<0.05$

$\mathrm{P}_{\mathrm{FDR}}<0.05$

$P_{\text {unc. }}<0.05$

B. Offset vs. no offset. CP showed enhanced emotional but decreased descending modulatory and reward networks. $(\mathbf{P}<\mathbf{0 . 0 1})$
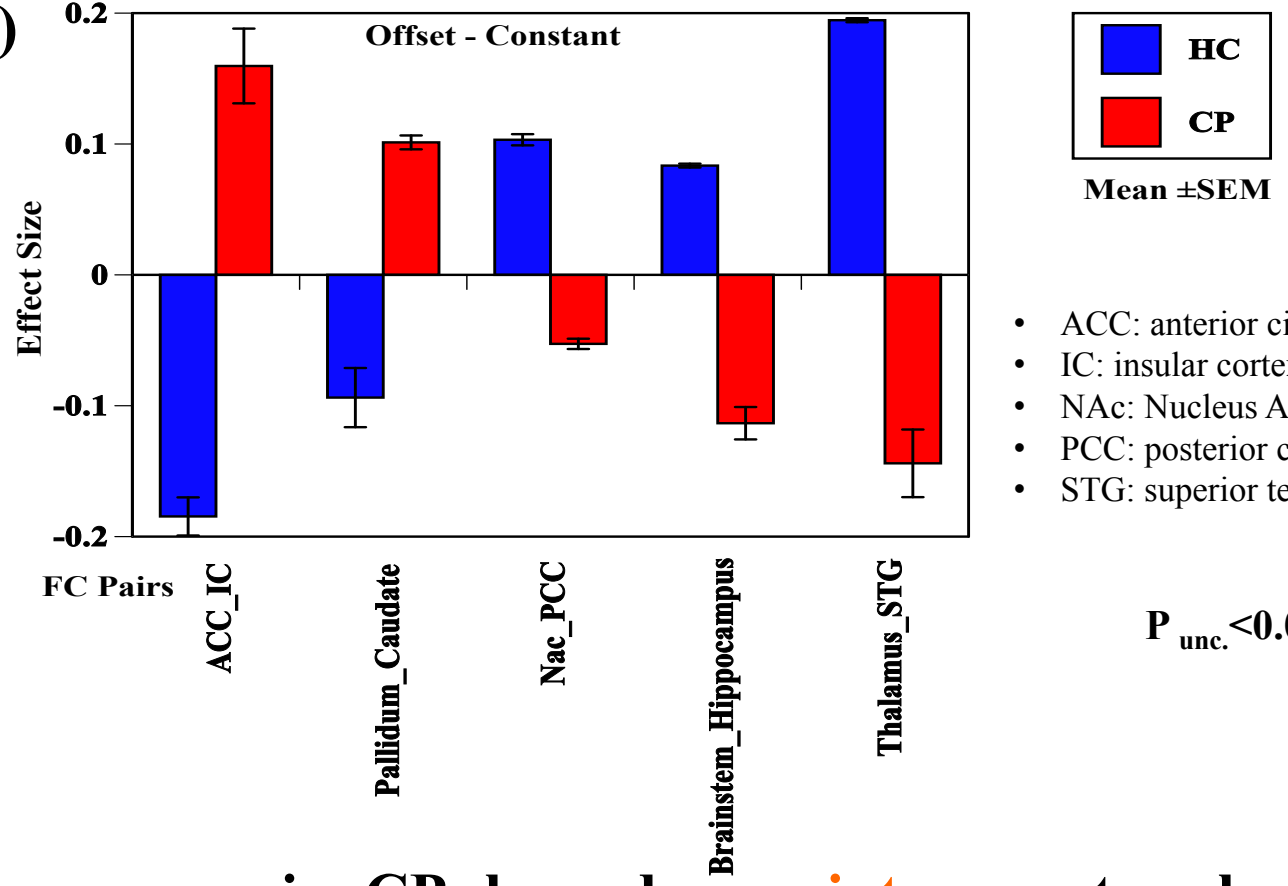

C. Pain vs. no pain. CP showed more intense network activities across emotion, descending modulatory, and reward networks. $(\mathbf{P}<\mathbf{0 . 0 1})$
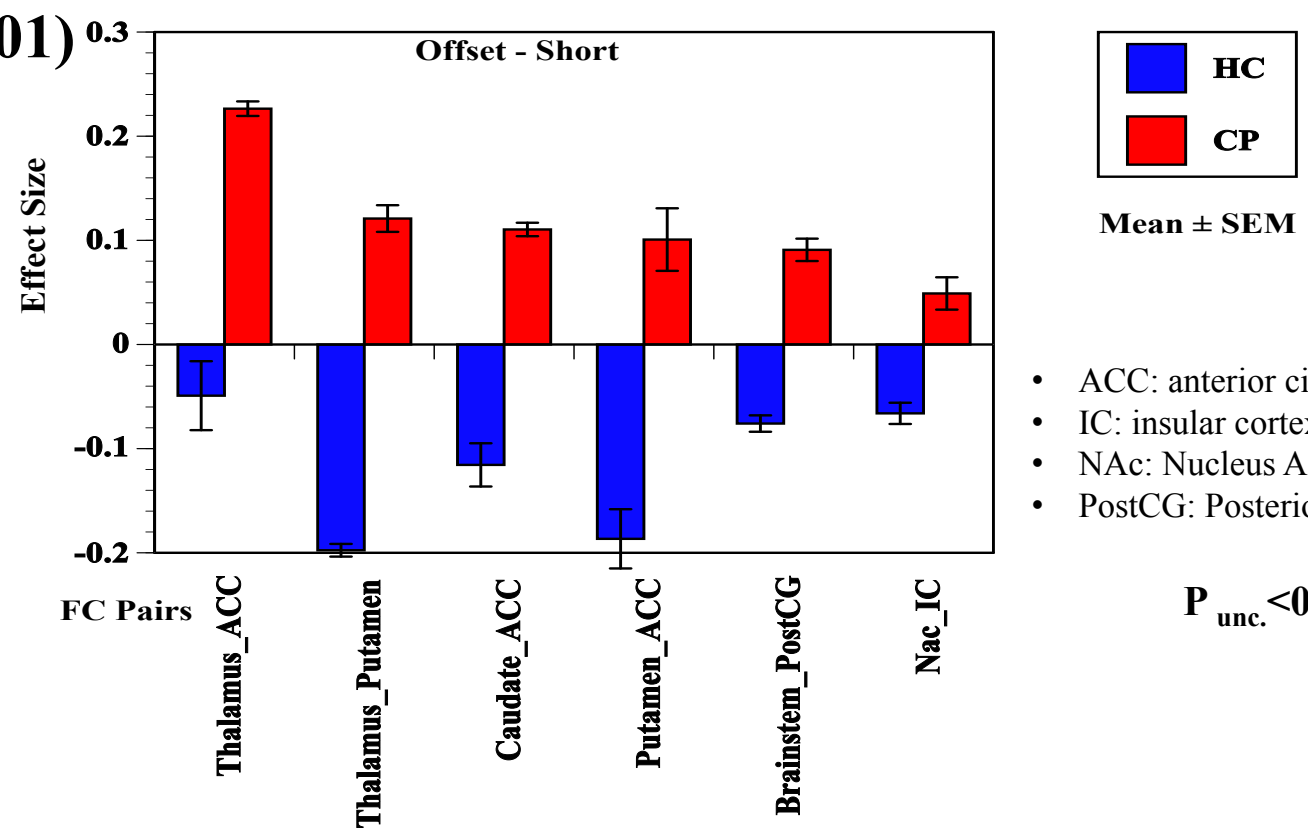

ACC: anterior cingulate cortex IC: insular cortex NAc: Nucleus Accumbens PostCG: Posterior cingulate gyrus

$P_{\text {unc. }}<0.01$

D. NAc-IC (=reward system) FC showed negative correlations with multiple scores of pain intensity, emotion, and cognition. $(\mathbf{P}<\mathbf{0 . 0 5})$
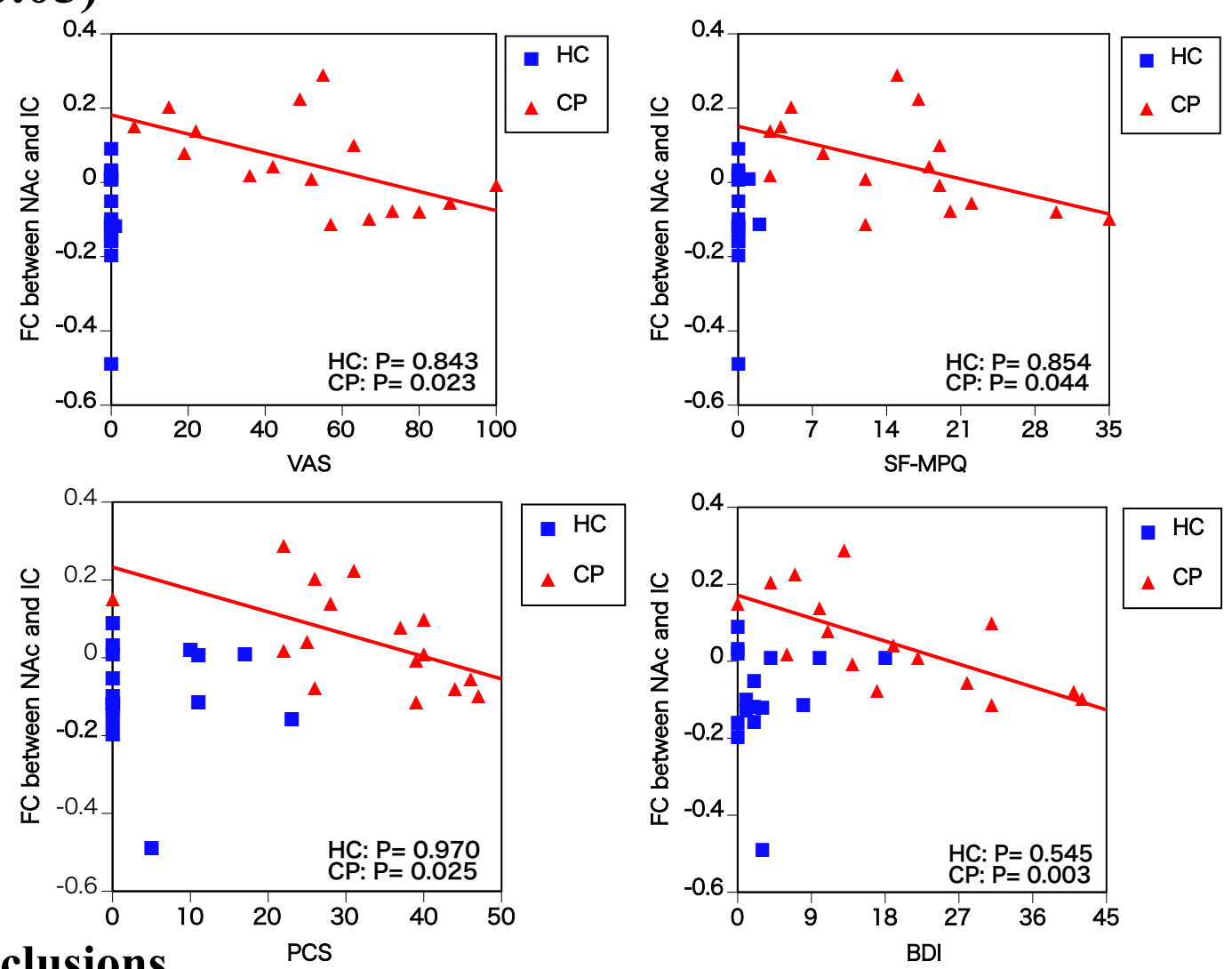

The present findings imply that chronic pain involves:

1) Default mode network weakness;

2) Emotional network hyperactivity;

3) "Pain matrix" exaggeration;

4) Descending pain modulatory and reward system dysfunctio 